\title{
Editorial
}

\section{Drying Modeling and Simulation}

\author{
Zhijun Zhang, ${ }^{1}$ Ireneusz Zbicinski, ${ }^{2}$ Lixin Huang, ${ }^{3}$ \\ Tsutomu Nozaki, ${ }^{4}$ and Zhonghua $\mathrm{Wu}^{5}$ \\ ${ }^{1}$ School of Mechanical Engineering and Automation, Northeastern University, Shenyang 110004, China \\ ${ }^{2}$ Faculty of Process and Environmental Engineering, Technical University of Lodz, 90-924 Lodz, Poland \\ ${ }^{3}$ Institute of Chemical Industry of Forest Products, Chinese Academy of Forestry, No. 16 Suojin 5th Village, \\ Nanjin 210042, China \\ ${ }^{4}$ Department of Environment Engineering, Kagoshima University, Korimoto 1-21-24, \\ Kagoshima 890-8580, Japan \\ ${ }^{5}$ Institute of Drying and Dewatering, College of Mechanical Engineering, Tianjin University of Science \\ and Technology, 1038 Daguan Road, Hexi District, Tianjin 300222, China
}

Correspondence should be addressed to Zhijun Zhang, zhjzhang@mail.neu.edu.cn

Received 19 June 2012; Accepted 19 June 2012

Copyright (C) 2012 Zhijun Zhang et al. This is an open access article distributed under the Creative Commons Attribution License, which permits unrestricted use, distribution, and reproduction in any medium, provided the original work is properly cited.

The idea behind this special issue of Mathematical Problems in Engineering is to consider the study and applications of modeling and simulation in drying technology. Drying science has a long history and is not only related to the food processing industry but also to a broad range of sectors such as chemistry, biochemistry, pharmaceuticals, and agriculture. The drying of various materials at different conditions in a wide variety of industrial and technological applications is a necessary step to obtain products that serve people's daily needs, to facilitate and enhance certain chemical reactions in engineering processes, or to make these processes environmentally safe.

As drying processes consume large amounts of energy, any improvement in the existing dryer design and any reduction in cost will be beneficial to the industry. Drying is inherently a cross-disciplinary and multidisciplinary process because it requires the optimal fusion of transport phenomena and materials science to supply heat, remove moisture from the material, and to generate a dehydrated product of specific quality.

The modeling and simulation of the drying process is a significant topic. This special issue contains nine papers, the contents of which are summarized as follows.

"Municipal sewage sludge drying treatment by a composite modifier" by N. Wei. Drying experiments of municipal sewage sludge were carried out to decrease the moisture content of sewage sludge. The effect of SCM components on sludge moisture content was analyzed using uniform design, and the optimum composition of SCM was determined through computer-aided modeling and optimization. 
"Thin-layer drying characteristics and modeling of Chinese jujubes" by X. Yi et al. A mathematical modeling of the thin-layer drying of jujubes in a convective dryer was established under controlled temperature and velocity conditions. The data from the experimental drying of jujubes were used to fit ten different thin-layer models. Then, the drying rate constants and the coefficients of the models tested were determined via nonlinear regression analysis using a statistical computer program.

"Simulation for sludge flocculation I: Brownian dynamic simulation for Perikinetic flocculation of charged particles" by L. Liu et al. To investigate the sludge drying process, a numerical simulation based on Brownian dynamic for the floc with uncharged and charged particles was conducted. The Langevin equation was used as a dynamical equation for tracking each particle in a floc. An initial condition and periodic boundary condition that conformed well to reality was used in calculating the floc growth process.

"Experimental study on forecasting mathematical model of drying shrinkage of recycled aggregate concrete" by Y. Guo and X. Wang. Based on the basic law in the AASHTO2007 model, the forecasting mathematical model for the drying shrinkage of recycled aggregate concrete was established through regression analysis and experimental study.

"SDSim: a novel simulator for solar drying processes" by Y. Bolea et al. SDSim is a novel solar dryer simulator based on a multicrop, inclined multipass solar air heater with in-built thermal storage mathematical model. SDSim has been developed as a design and development tool used to study and forecast the behavior of the system model to improve its drying efficiency and achieve a return on investment.

"Model of mass and heat transfer during vacuum freeze-drying for cornea" by $\mathrm{H}$. Zou et al. A theoretical analysis of corneal freeze-drying was presented, and a mathematical model for describing heat and mass transfer during corneal vacuum freeze-drying was established. The analogy computation for the freeze drying of cornea was done using finite-element computational software.

"Distribution of vapor pressure in the vacuum freeze drying equipment" by S. Zhang and J. Liu. A mathematical model was developed to describe the vapor flow in the passageways either between material plates or in the channel between plate groups. The distribution of vapor pressure along the flow passageway was described. Two characteristic factors of passageways were defined to express the effects of structural and process parameters on vapor pressure distribution.

"Nonequilibrium thermal dynamic modeling of porous medium vacuum drying process" by Z. Zhang and N. Kong. Based on the theory of heat and mass transfer, a coupled model for vacuum drying of a porous medium was constructed. The model was implemented and solved with COMSOL software. The water evaporation rate was determined via the nonequilibrium method using the rate constant parameter.

"Model predictive control of the gain drying process" by F. Han et al. Using mathematical models and the quality model of the corn drying process, a digital simulation of the corn drying machine system based on a virtual instrument was established for the 5HSZ dryer. The device could automatically control the air temperature and predict the discharge speed of grain.

\section{Acknowledgments}

The authors are grateful to the authors of the special issue for their contributions. They thank the reviewers for their valuable comments on the submissions. They highly appreciate 
the support from the editorial members of Mathematical Problems in Engineering, as well as the editorial staff of Hindawi Publishing Corporation. Dr. Zhang acknowledges the support from the National Natural Science Foundation of China (Grant nos. 31000665 and 51176027).

Zhijun Zhang

Ireneusz Zbicinski

Lixin Huang

Tsutomu Nozaki

Zhonghua Wu 


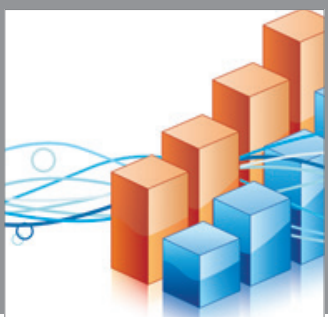

Advances in

Operations Research

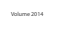

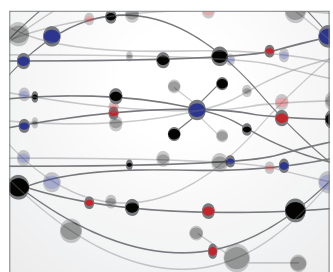

\section{The Scientific} World Journal
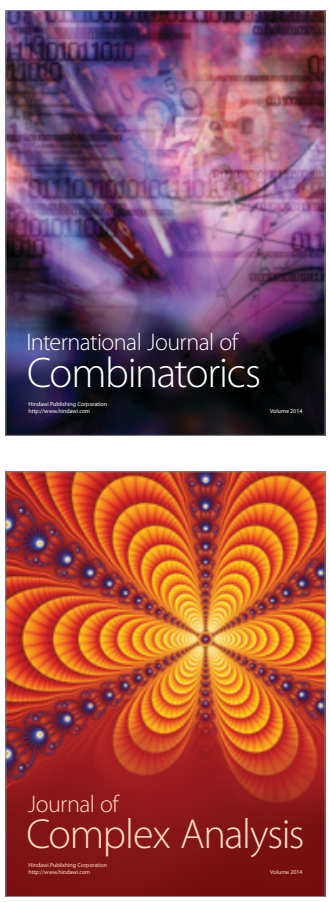

International Journal of

Mathematics and

Mathematical

Sciences
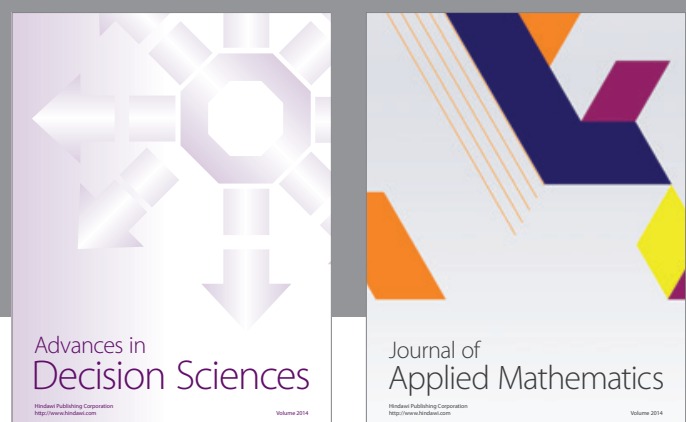

Journal of

Applied Mathematics
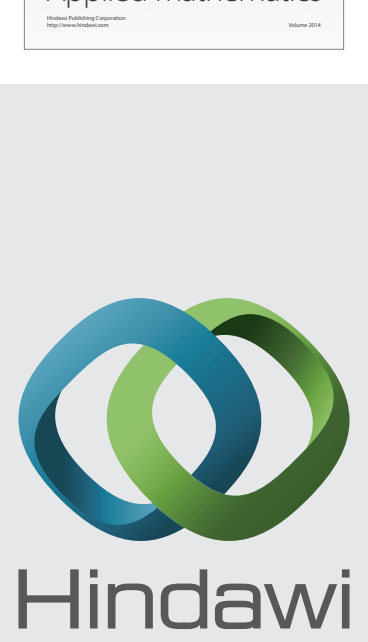

Submit your manuscripts at http://www.hindawi.com
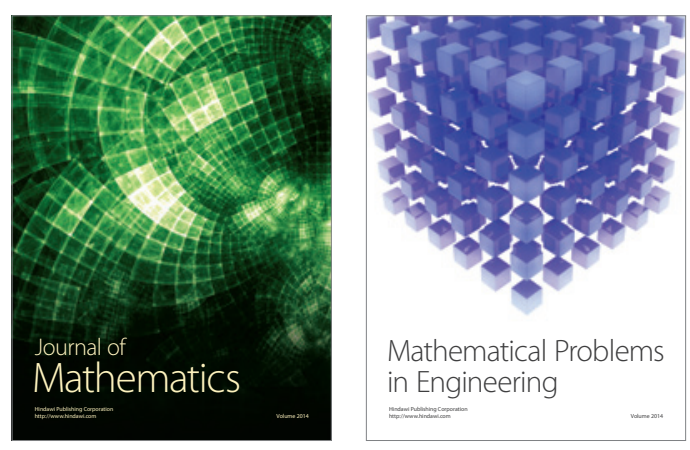

Mathematical Problems in Engineering
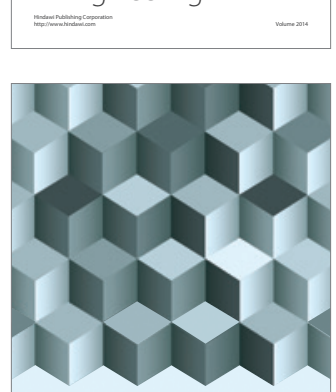

Journal of

Function Spaces
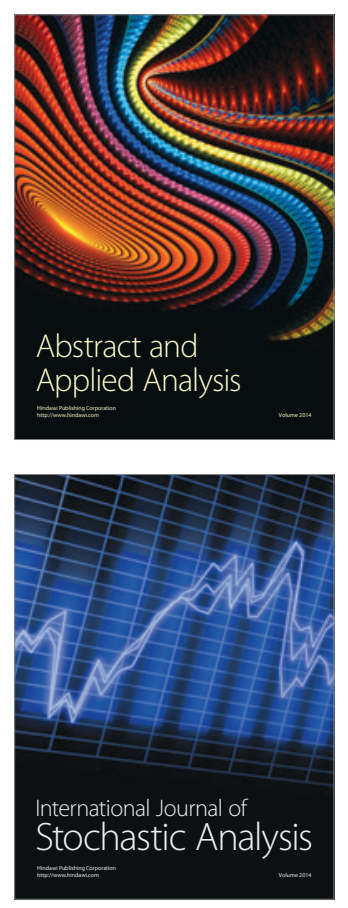

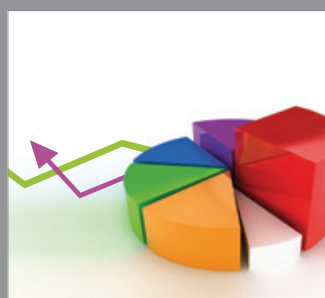

ournal of

Probability and Statistics

Promensencen
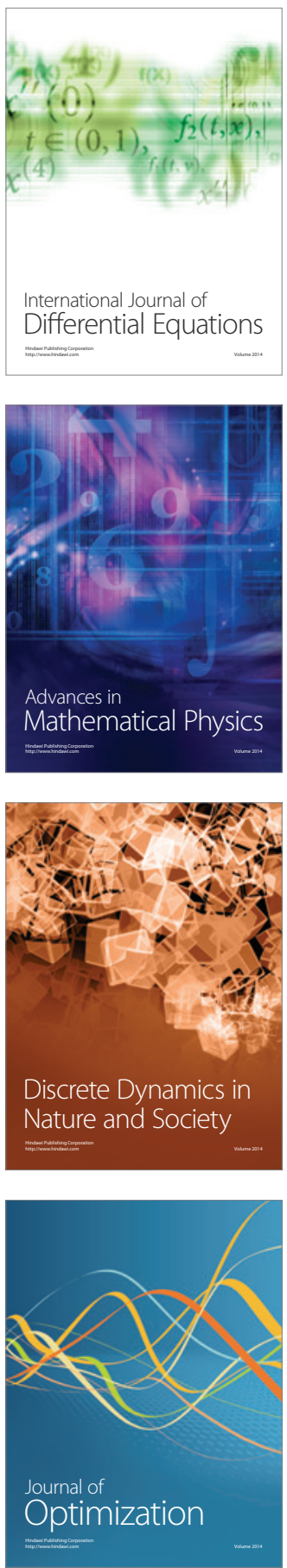